\title{
Can diffusion- and perfusion-weighted imaging alone accurately triage anterior circulation acute ischemic stroke patients to endovascular therapy?
}

\author{
Dylan N Wolman, ${ }^{1}$ Michael Iv, ${ }^{1}$ Max Wintermark, ${ }^{1}$ Gregory Zaharchuk, ${ }^{1}$ \\ Michael P Marks, ${ }^{1}$ Huy M Do, ${ }^{1}$ Robert L Dodd ${ }^{2}$ Gregory W Albers, ${ }^{3}$ \\ Maarten $G$ Lansberg, ${ }^{3}$ Jeremy J Heit ${ }^{1}$
}

${ }^{1}$ Department of Radiology, Neuroimaging and Neurointervention Division, Stanford University Medical Center, Stanford, California, USA ${ }^{2}$ Department of Neurosurgery, Stanford University Medical Center, Stanford, California, USA ${ }^{3}$ Department of Neurology, Stanford Stroke Center, Stanford University Medical Center, Stanford, California, USA

\section{Correspondence to} Dr Jeremy J Heit, Department of Radiology, Neuroimaging and Neurointervention Division, Stanford University Medical Center, Stanford, CA 94305, USA; jheit@stanford.edu

Received 22 January 2018 Revised 24 February 2018 Accepted 28 February 2018 Published Online First 19 March 2018
Check for updates

To cite: Wolman DN, Iv M Wintermark $\mathrm{M}$, et al. $J$ Neurolntervent Surg 2018:10:1132-1136.

\begin{abstract}
Background and purpose Acute ischemic stroke (AIS) patients who benefit from endovascular treatment have a large vessel occlusion (LVO), small core infarction, and salvageable brain. We determined if diffusion-weighted imaging (DWI) and perfusion-weighted imaging (PWI) alone can correctly identify and localize anterior circulation LVO and accurately triage patients to endovascular thrombectomy (ET).
\end{abstract}

Materials and methods This retrospective cohort study included patients undergoing MRI for the evaluation of AIS symptoms. DWI and PWI images alone were anonymized and scored for cerebral infarction, LVO presence and LVO location, DWI-PWI mismatch, and ET candidacy. Readers were blinded to clinical data. The primary outcome measure was accurate ET triage. Secondary outcomes were detection of LVO and LVO location.

Results Two hundred and nineteen patients were included. Seventy-three patients (33\%) underwent endovascular AIS treatment. Readers correctly and concordantly triaged 70 of 73 patients $(96 \%)$ to $\mathrm{ET}(\kappa=0.938 ; \mathrm{P}=0.855)$ and correctly excluded 143 of 146 patients (98\%; $P=0.942)$. DWI and PWI alone had a $95.9 \%$ sensitivity and a $98.4 \%$ specificity for accurate endovascular triage. LVO were accurately localized to the ICA/M1 segment in 65 of 68 patients (96\%; $\kappa=0.922 ; P=0.817)$ and the $M 2$ segment in 18 of 20 patients $(90 \% ; \kappa=0.830 ; P=0.529)$.

Conclusion AIS patients with anterior circulation LVO are accurately identified using DWI and PWI alone, and LVO location may be correctly inferred from PWI. MRA omission may be considered to expedite AIS triage in hyperacute scenarios or may confidently supplant non-diagnostic or artifact-limited MRA.

\section{INTRODUCTION}

Approximately 35\% of acute ischemic stroke (AIS) is due to large vessel occlusion (LVO) of the internal carotid artery (ICA) or M1 and M2 segments of the middle cerebral artery (MCA). ${ }^{1}$ AIS due to LVO may be treated with endovascular thrombectomy (ET), which results in significantly improved clinical outcomes relative to medical therapy. ${ }^{2}$ ET is particularly effective when delays to reperfusion are minimized. ${ }^{3}$

AIS patient triage to ET requires rapid neuroimaging to identify optimal candidates and to exclude patients with contraindications to treatment.
Endovascular candidates most likely to benefit from treatment have: a small core infarction; salvageable brain tissue; and large vessel occlusion (LVO). ${ }^{4}$ Current treatment guidelines recommend that AIS patients undergo non-invasive neuroimaging triage before ET, which minimally includes a non-contrast head CT and CTA. ${ }^{56}$ MRI remains the gold standard in the assessment of cerebral ischemia and has been demonstrated to be both safe and efficacious in the triage of AIS patients. ${ }^{7-9}$ MRI may be particularly useful in the evaluation of AIS patients who present in late time windows ${ }^{10}{ }^{11}$ and is extensively utilized in concert with PWI in recent trials expanding the time window for ET. ${ }^{12} 13$

AIS patient selection for ET on the basis of a DWI-PWI mismatch is well established and correlated to improved functional and angiographic outcomes. ${ }^{8} 14$ Benefits of PWI include the identification of DWI-negative cerebral ischemia, matched perfusion deficits, and malignant perfusion profiles, all of which optimize patient selection for endovascular treatment. ${ }^{15}$ Additionally, PWI provides an indirect quantitative measurement of collateral vessel status, ${ }^{16}$ which is an established predictor of favorable outcomes following AIS. ${ }^{18}$

All AIS neuroimaging protocols seek a balance between providing detailed information for the optimal triage of patients to ET and minimizing the imaging time to facilitate expeditious treatment. ${ }^{3}$ Rapid AIS MRI protocols typically include DWI, PWI, MRA, and gradient-echo (GRE) sequences. ${ }^{19}$ Further reduction in the length of such an already streamlined MRI protocol requires the extraction of more information from fewer sequences. A detailed understanding of vascular territories may allow inference of the presence and location of a LVO from PWI data alone. ${ }^{20}$ We hypothesized that DWI and PWI sequences alone may be sufficient to triage patients with anterior circulation AIS symptoms to ET. We determined the accuracy of endovascular candidacy, LVO identification, and LVO localization using only DWI and PWI in the absence of any clinical data.

\section{MATERIALS AND METHODS}

\section{Study design}

The study was approved by our Institutional Review Board and complied with the Health Insurance Portability and Accountability Act. We performed a retrospective cohort study of all 73 consecutive 
patients who underwent AIS triage to ET with MRI at our neurovascular referral center between January 1, 2012 and December 31, 2016. All MRI studies included DWI, GRE, MRA, and PWI. All patients triaged to ET presented within 16 hours of symptom onset. We included an additional 146 consecutive patients who underwent MRI for the evaluation of acute cerebral ischemia. These patients had symptoms consistent with acute ischemic stroke due to LVO or a more distal occlusion, and this group was included as a control group to enable comparison between ET-eligible and ET-ineligible cases. In total, 219 patients were included in our study. Exclusion criteria included patients with posterior circulation LVO and MRI studies with excessive motion or technical failure of PWI.

Gold standard assessment of cerebral infarction, cerebral hemorrhage, LVO presence, LVO location, perfusion deficit (measured as the volume of tissue with a Time-to-maximum (Tmax) delay of $>6 \mathrm{~s}$ ), and DWI-PWI mismatch (defined as a mismatch between the DWI and Tmax $>6$ seconds volume of at least $15 \mathrm{~mL}$ and a mismatch ratio of 1.8 ) were determined from the official radiology report and independently validated by an experienced neuroradiologist.

Anterior circulation LVO was categorized as an occlusion of the ICA, M1, or M2 segment of the MCA. MCA vessel segments were defined on a strictly anatomic basis: M1 segment was the horizontal segment; M2 segments started at the vertical inflection overlying the insula and terminated at the circular sulcus; and M3 and M4 segments were distal to the circular sulcus inflection. Patients appropriate for ET had: a cerebral infarction volume estimated to be $\leq 70 \mathrm{~mL}$; a LVO (ICA, M1, or M2 MCA segment occlusion); a DWI-PWI mismatch; and absent cerebral hemorrhage. ${ }^{8}$

DWI and Tmax PWI maps from all patients were anonymized and entered into an Osirix (Pixmeo, Bernex, Switzerland) database for imaging review by three readers: an experienced diagnostic neuroradiologist; an experienced neurointerventional radiologist; and a radiology resident. Each reader visually scored DWI and Tmax images for: the presence of acute infarction; the presence of a LVO and, if present, LVO location (ICA, M1, or M2); PWI deficit on Tmax maps; DWI-PWI mismatch presence; the presence of any contraindication to ET (such as cerebral hemorrhage, matched DWI-PWI, or estimated core infarct volume $>70 \mathrm{~mL}$ ); and ET candidacy according to DEFUSE 2 criteria. ${ }^{8}$ Automatically quantified Tmax delay volumes on the RAPID maps were not utilized by readers, and DWI volumes were only utilized if visual estimation of the core infarct volume was thought to be borderline. Readers were blinded to MRA and GRE sequences, clinical data (including time from symptom onset and National Institutes of Health Stroke Scale (NIHSS)), and any prior imaging studies. Clinical data were intentionally excluded from analysis to isolate the predictive capabilities of PWI/DWI in ET triage. The accuracy of individual reader scores was determined by comparison to final MRI and angiography reports.

The primary outcome measure was accurate ET triage using DWI and PWI alone compared with ET triage by the neurointerventionalist at the time of presentation. Secondary outcome measures were accurate LVO identification and LVO localization compared with MRA interpretation by experienced neuroradiologists from the original MRA reports.

\section{Neuroimaging protocol details}

All included patients underwent stroke protocol MRI, which minimally included DWI, GRE, time-of-flight head MRA, and bolus PWI with automatic image post-processing by RAPID software (iSchemaView, Menlo Park, California). ${ }^{21}$ Our institution utilizes a rapid stroke protocol that includes SSFSE localization images (0:20 min), DWI (0:41 min), GRE (1:56 min), PWI (1:48 min), and time-of-flight head MRA (2:25 min), for a total imaging time of 7:10 min. These MRI protocols for AIS triage to ET do not include the aortic arch or cervical vascular imaging. Imaging was performed on a 1.5T GE Signa or 3.0T GE MR750 MRI scanner using an eight-channel GE HR brain coil (GE Healthcare, Milwaukee, WI). Technical imaging parameters were as follows: DWI parameters: $\mathrm{TR}=6000 \mathrm{msec}$, $\mathrm{TE}=78.2 \mathrm{msec} ; \mathrm{b}$-value $=0$ and 1000 ; flip angle $90^{\circ}$, and PWI parameters: $\mathrm{TR}=1800 \mathrm{msec}, \mathrm{TE}=35 \mathrm{msec}$; flip angle $80^{\circ}$. All images were acquired with $5 \mathrm{~mm}$ slice thickness. Dynamic phase bolus perfusion imaging was performed following the intravenous administration of Multihance (Bracco, Milan, Italy) into an antecubital vein at a rate of $4.0 \mathrm{~mL} / \mathrm{sec}$ using a power injector. Imaging coverage extended from the vertex to the occiput.

\section{Statistical analysis}

For each criterion scored per reader, comparisons against reference data were analyzed using a two-tailed chi-squared test $(\alpha=0.05)$. Inter-reader agreement was assessed using Fleiss' kappa test. Statistical significance was set at the 0.05 level. Individual reader scores were pooled by average for analysis with consensus between at least two of three readers. Pooled reader accuracy was determined by a $\mathrm{P}$ value $>0.05$, demonstrating a lack of a significant difference from reference data. All data were processed using Excel (Microsoft, Redmond, WA).

\section{RESULTS}

\section{Imaging triage and candidacy for endovascular therapy}

Two hundred and nineteen patients (49\% female, mean age 66.4 years) met our inclusion criteria. Seventy-three patients (33\%) underwent endovascular AIS treatment following MRI evaluation. The remaining 146 patients did not undergo endovascular AIS treatment due to a matched perfusion deficit (11 patients, 7.7\%), cerebral hemorrhage (15 patients, 10.3\%), clinical inappropriateness for treatment (one patient, $0.7 \%$ ), or absence of cerebral ischemia and/or LVO (131 patients, 89.7\%).

Quantitated core infarct, Tmax delay $>6 \mathrm{~s}$, and mismatch volumes were calculated by RAPID in a post hoc analysis after the study was completed and are summarized in online supplementary table 1.

When anonymized DWI and PWI sequences were reviewed, readers accurately identified the presence of acute infarction in 113 of 118 patients $(96 \% ; \kappa=0.926 ; \mathrm{P}=0.676), \mathrm{LVO}$ in 86 of 88 patients $(98 \% ; \kappa=0.943 ; \mathrm{P}=0.921)$, and a DWI-PWI mismatch in 76 of 79 patients $(96 \% ; \kappa=0.921 ; \mathrm{P}=0.825)$. Among all patients with AIS due to an anterior circulation LVO, 2 of 88 patients $(2.3 \%)$ were not identified by DWI and PWI alone.

Readers correctly and concordantly triaged 70 of 73 patients $(96 \%)$ to ET $(\kappa=0.938 ; \mathrm{P}=0.855)$ and correctly excluded 143 of 146 patients $(98 \% ; \mathrm{P}=0.942)$ from ET (Table 1$)$. Therefore, DWI and PWI alone have a sensitivity of $95.9 \%$ and a specificity of $98.4 \%$ for accurate ET triage (table 1). False negative exclusion from ET was determined on the basis of even a single discordant reader, as was the case for false positive inclusion of control patients in ET. Two of the three patients incorrectly excluded from ET had cervical carotid stenoses and fluctuating neurologic symptoms, but readers were blinded to clinical data. The third erroneously excluded patient had a high presenting NIHSS score of 25 and a borderline perfusion mismatch of exactly $15 \mathrm{~mL}$. Post-processed quantitative maps of core infarction and Tmax 
Table 1 Endovascular candidacy and triage

\begin{tabular}{|c|c|c|c|c|c|c|c|c|}
\hline & \multicolumn{2}{|l|}{ Overall } & \multicolumn{3}{|l|}{ Positive cases } & \multirow[b]{2}{*}{$\begin{array}{l}\text { Inter-reader } \\
\text { agreement }\end{array}$} & \multirow[b]{2}{*}{ Sensitivity } & \multirow[b]{2}{*}{ Specificity } \\
\hline & Correct & Incorrect & Correct & Incorrect & $\begin{array}{l}\text { Reference } \\
\text { agreement }\end{array}$ & & & \\
\hline Acute infarction & $208 / 219(95.1 \%)$ & $11 / 219(4.9 \%)$ & $113 / 118(95.5 \%)$ & $5 / 118(4.5 \%)$ & $P=0.676$ & $\mathrm{~K}=0.926$ & . & . \\
\hline Large vessel occlusion & $216 / 219(98.5 \%)$ & $3 / 219(1.5 \%)$ & $86 / 88(97.7 \%)$ & $2 / 88(2.3 \%)$ & $P=0.921$ & $\mathrm{~K}=0.943$ & . & . \\
\hline $\begin{array}{l}\text { Diffusion-perfusion } \\
\text { mismatch }\end{array}$ & $212 / 219(96.7 \%)$ & $8 / 219(3.7 \%)$ & $76 / 79(96.2 \%)$ & $3 / 79(3.8 \%)$ & $P=0.825$ & $\mathrm{~K}=0.921$ & . & . \\
\hline $\begin{array}{l}\text { Triage to endovascular } \\
\text { therapy }\end{array}$ & $214 / 219(97.6 \%)$ & $5 / 219(2.4 \%)$ & $70 / 73(95.9 \%)$ & $3 / 73(4.1 \%)$ & $P=0.855$ & $\mathrm{~K}=0.938$ & $95.9 \%$ & $98.4 \%$ \\
\hline
\end{tabular}

values better demonstrated this small mismatch, but blinding of readers to clinical data accounted for the erroneous exclusion of this patient. Three of 146 patients (2\%) from the control group were erroneously triaged to ET, two by two readers each and the third by all three readers. One of these two patients demonstrated cerebral hemorrhage which was not recognized by readers in the absence of a GRE sequence, while the other demonstrated a borderline perfusion mismatch considered too small for intervention at the time of triage. All three readers erroneously triaged the third patient to ET due to a large territorial perfusion mismatch. However, the patient was of advanced age and demonstrated a malignant perfusion pattern and was therefore deemed a poor ET candidate.

\section{Large-vessel occlusion localization}

Readers accurately localized vessel occlusions to the ICA/ M1 segments in 65 of 68 patients $(96 \% ; \kappa=0.922 ; \mathrm{P}=0.817$; table 2 ; figure $1 \mathrm{~A}-\mathrm{B})$, which were pooled together given the similar appearance of perfusion deficits caused by these LVOs (figure 2). Accurate localization of M2 segment vascular occlusions occurred in 18 of 20 patients $(88 \% ; \kappa=0.830 ; \mathrm{P}=0.529$; table 2). M2 misclassifications were most frequently localized to the M1 segment (mean 2 of 20 patients (10\%); range $0-4$ $(0-20 \%) \%$ ] by reader; figure $1 \mathrm{C})$. Failure to identify a LVO by any individual reader occurred in 0-2 patients with ICA LVO (0\%-13\%), 1-2 patients with M1 LVO (2\%-4\%), and 0-1 patient with M2 LVO (0\%-5\%; figure $1 \mathrm{~A}-\mathrm{C}$, table $2 \mathrm{~B})$.

The absence of a LVO was accurately classified (mean 130/131 [99\%]; $\kappa=0.937 ; \mathrm{P}=0.977$; table 2; figure $1 \mathrm{D}$, table $2 \mathrm{~B}$ ). One patient without a LVO was misclassified with an ICA occlusion by two readers ( 1 of 131 patients $(0.8 \%)$; figure $1 \mathrm{D}$, table $2 \mathrm{~B}$ ). This patient had high-grade atherosclerotic stenosis of the supraclinoid left ICA and presented with confluent infarction and a large area of oligemia in the left ICA territory. The readers mis-interpreted the oligemia as tissue-at-risk of infarction and incorrectly triaged the patient to endovascular treatment. This mistake would likely have been avoided if the readers had access to $\mathrm{CBV}$ images, which demonstrated increased CBV that was consistent with a chronic proximal stenosis, automated Tmax quantification, which demonstrated a volume of Tmax $>6 \mathrm{~s}$ of only $4.3 \mathrm{~mL}$, or clinical data, which demonstrated a low NIHSS in this patient.

\section{DISCUSSION}

In this study, we found DWI and PWI sequences alone to be highly accurate in the triage of anterior circulation AIS patients to ET. Our readers accurately and concordantly triaged $96 \%$ of patients to ET using only DWI and PWI sequences in the absence of clinical information. The three patients who were incorrectly excluded from endovascular therapy in our study had subtle imaging findings of AIS and LVO, which were not accurately interpreted by our readers. However, these three patients had neurologic symptoms consistent with AIS due to LVO, and we expect that the inclusion of these clinical data, as would occur outside of a study designed to isolate the predictive value of imaging alone, would result in more accurate triage following interpretation of DWI and PWI in these patients. Specifically, we posit that inclusion of clinical data in this study would actually confound our results, as it would be impossible to separate the weight placed by readers on the clinical versus imaging data when considering ET triage.

We also hypothesized that cerebral perfusion deficit patterns identified by PWI are specific to the causative vascular occlusion, ${ }^{20} 22$ and that the presence of a LVO may be inferred from PWI alone. We found that anterior circulation LVO may be accurately localized using PWI alone in up to $96 \%$ of patients. Our results suggest that PWI may be used to infer the presence of anterior circulation LVO with a high degree of confidence, which may be beneficial in troubleshooting artifact-degraded, motion-degraded, or non-diagnostic MRA studies. Stated differently, patients with a LVO and a small core infarction who underwent ET have a large perfusion deficit. By contrast, patients who did not undergo ET had a LVO, a large core infarction, and no significant perfusion deficit (online supplementary table 1). Therefore, our findings are in good agreement with previously established methods of triage set forth by the SWIFT-PRIME, EXTEND-IA, and DEFUSE 3 trials. ${ }^{13} 2324$

Neuroimaging protocols for AIS triage vary widely by institution, and controversy remains regarding whether MRI over CT is the most optimal for ET triage. ${ }^{25}$ DWI remains the gold standard in the assessment of cerebral ischemia, and MRI has demonstrated safety and efficacy in the identification of appropriate ET candidates and, at our institution, is performed as rapidly as CT-based triage. ${ }^{781519}$ Some institutions have recommended complex combined workflows utilizing both CT and MRI in AIS triage. ${ }^{25}$ However, such protocols require a highly streamlined process that is impractical for many institutions due to physically separate locations of CT and MRI scanners and the necessity of holding both a CT-scanner and an MRI open for the evaluation of a single patient.

The high sensitivity and specificity of DWI and PWI sequences alone for ET triage suggests that an ultra-rapid MRI protocol that uses PWI rather than MRA for the determination of LVO presence may be adopted, or that MRA may be confidently supplanted if unavailable or artifact-degraded. Our institution uses a rapid stroke protocol that includes localization images (0:20 min), DWI (0:41 min), GRE (1:56 min), PWI (1:48 min), and time-of-flight head MRA (2:25 min). Omission of MRA in an ultra-rapid MRI protocol would reduce the total scan time by approximately $2.5 \mathrm{~min}(34 \%)$ from $7: 10 \mathrm{~min}$ to $4: 45 \mathrm{~min}$. 


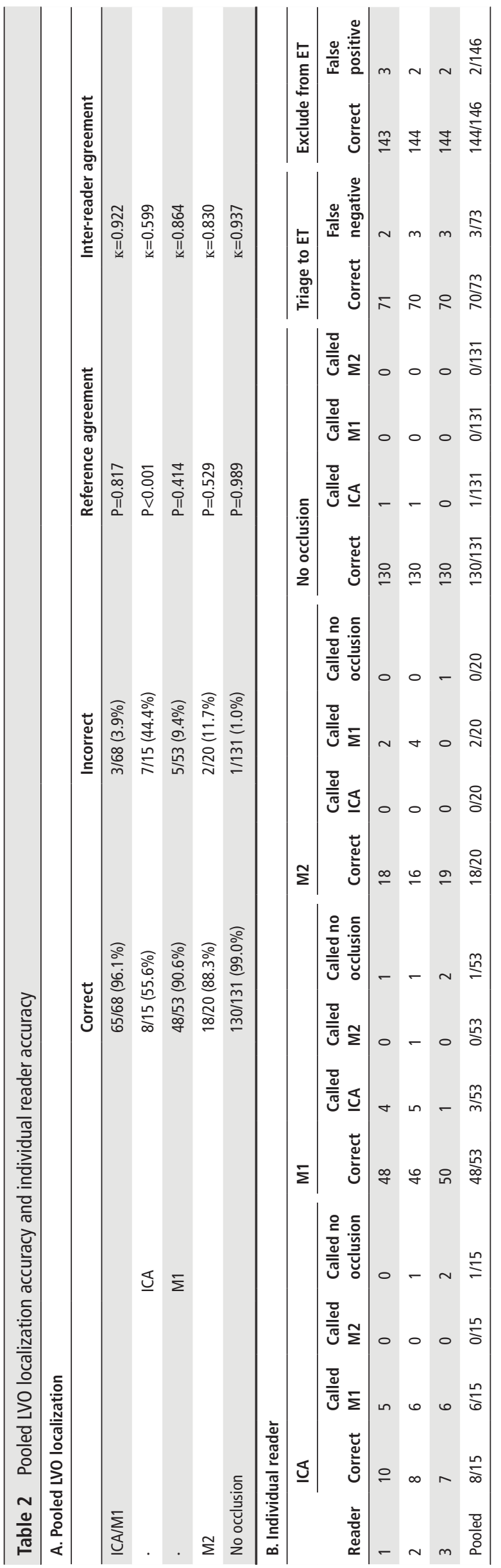

While this modest reduction in scan time may have a small but measurable impact on patient outcome,${ }^{26}$ the systematic saving of minutes during AIS patient triage to endovascular treatment may have a larger impact on outcome in patients presenting in the hyperacute period, particularly when those with poor collaterals are more likely to have salvageable brain tissue. In support of the idea that incremental time savings are beneficial, other studies have estimated that each minute of delay to revascularization results in a $0.3 \%$ reduction in the probability of achieving a good functional outcome, ${ }^{2728}$ and that reduced time to revascularization remains a critical predictor of improved outcomes. ${ }^{3}$

Following patient triage to ET, the absence of non-invasive cerebral, cervical, or aortic vessel imaging is unlikely to affect the neurointerventional workflow. Most neurointerventionalists perform ET with standardized access constructs and equipment that are designed to rapidly navigate a wide range of complex arterial anatomy during ET procedures. While some institutions acquire non-invasive aortocervical vascular imaging for all patients, we do not routinely evaluate the aortocervical vasculature at our institution. We have not identified any procedural detriment due to our more selective imaging, and our institutional groin puncture to revascularization times are superior to those reported in recent randomized trials (Wolman and Heit, unpublished data). ${ }^{29}$

There is evidence that PWI (CT or MRI) is superior for the evaluation of AIS due to LVO presenting in late time windows. ${ }^{10} 11$ The DAWN (DWI or CTP Assessment With Clinical Mismatch in the Triage of Wake Up and Late Presenting Strokes Undergoing Neurointervention) ${ }^{12}$ and DEFUSE 3 (Endovascular Therapy Following Imaging Evaluation for Ischemic Stroke 3$)^{13}$ trials utilized a PWI-based patient selection methodology to randomize late-time window AIS patients to either ET or medical therapy. Both studies were halted early after an interim analysis demonstrated a significant benefit for patients treated with ET. Results of the DAWN and DEFUSE 3 trials are expected to markedly increase the number of patients eligible for ET treatment triage using PWI, which has the potential to overwhelm comprehensive and neurointerventional capable stroke centers. Improved imaging triage of AIS patients prior to transfer to comprehensive centers will likely be required to efficiently evaluate these late time window patients.

Currently, as few as $28 \%$ of AIS patients transferred to comprehensive stroke centers for ET undergo treatment, and the routine use of vascular imaging at referring hospitals has the potential to prevent up to $20 \%$ of unnecessary patient transfers. ${ }^{30}$ The results of our study are readily adaptable to CT PWI techniques, and the use of ultra-rapid AIS imaging evaluation using PWI (CT or MRI) may be employed at referring hospitals to more effectively triage patients who are likely to benefit from ET to comprehensive and neurointerventional capable hospitals. Particular consideration should be given to the broad adoption of PWI with automated post-processing at referring hospitals given that both the DAWN and DEFUSE 3 trials used this technique to determine ET candidacy, which will likely establish PWI as a necessary component of standard-of-care neuroimaging for AIS. We expect that neurointerventionalists will benefit from knowing that a late time-window AIS patient who is undergoing transfer for possible ET meets DAWN and/or DEFUSE 3 treatment criteria.

Community hospitals may have reduced access to experienced neuroradiologists and neurologists experienced in interpreting advanced brain imaging, such as CTA and MRA. Our study demonstrated a similar degree of accuracy in image interpretation between an experienced neuroradiologist, an experienced neurointerventional radiologist, and a first-year radiology 


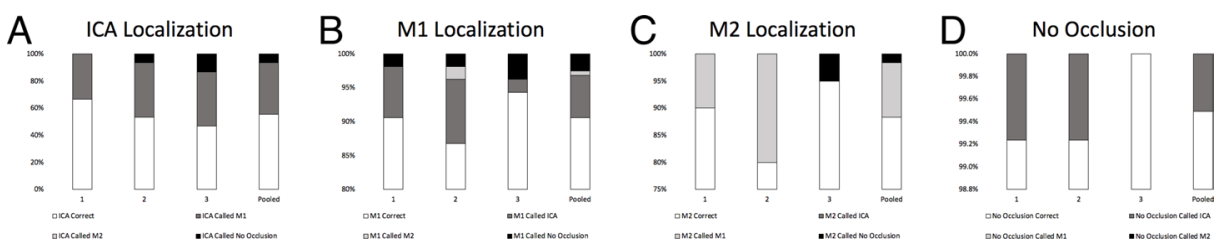

Figure 1 Individual LVO localization and triage accuracy. Individual reader accuracy in the localization of LVOs to specific vessel segments: (A) ICA; (B) M1; (C) M2; and (D) no occlusion. Each reader is numbered 1-3, with pooled reader accuracy displayed as a fourth bar in each graph. The bottom portion of each graph denotes accurate localization compared with reference reports, with different shaded segments above denoting the breakdown of corresponding localization errors. Note that the scale for each graph varies.

resident, which suggests that PWI interpretation at smaller hospitals less accustomed to advanced neuroimaging may remain highly accurate. Additional studies may test this hypothesis, and we have begun piloting this approach in the triage of AIS patients for transfer from our own spoke hospitals.

Our timely results may be used as a road map in establishing rapid and efficient neuroimaging protocols at referring hospitals that are likely to simplify and expedite the appropriate triage and transfer of AIS patients, while potentially reducing the costly transfer of patients who are ineligible for ET.

\section{Limitations}

Our study is limited by the retrospective and non-randomized study design, which may introduce bias. The study was performed predominantly by experienced neuroradiologists and neurointerventional radiologists, which may limit the generalizability of our findings. Differences in triage by the readers in our retrospective study when compared with the time of patient evaluation is undoubtedly influenced by clinical factors, physical examination findings, and additional MRI sequences (such as GRE) that were not provided to our readers, though this blinding was an intentional effort to experimentally isolate the value of imaging data for patient triage. Nonetheless, these differences may bias the results or

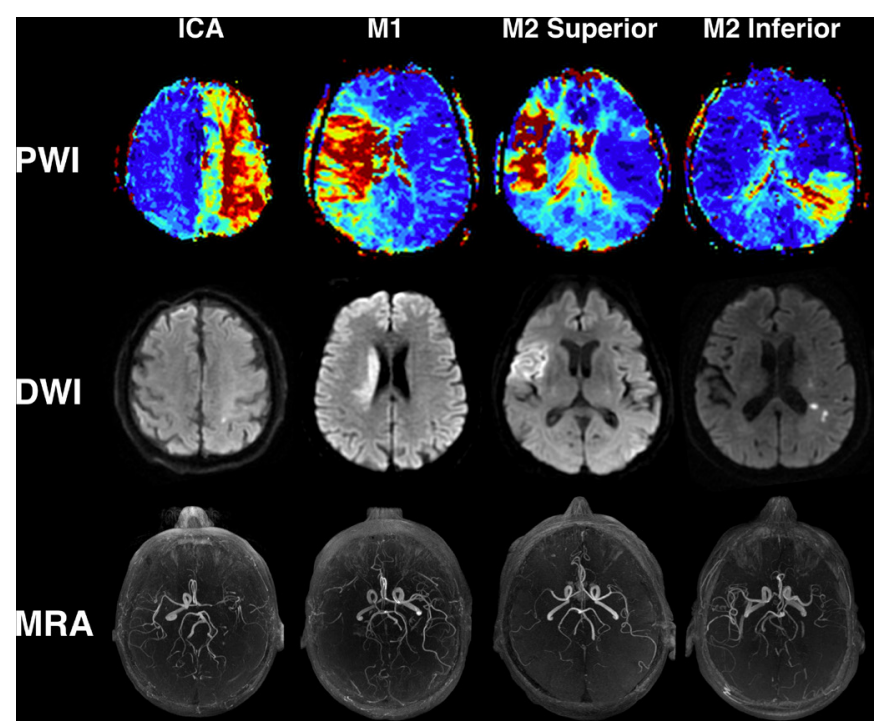

Figure 2 Perfusion patterns of large vessel occlusions. Sample perfusion patterns seen with specific territorial LVOs (upper row) with corresponding early pre-catheterization regions of infarction in each case (middle row) and focal occlusions demonstrated by MRA (lower row). Note that the perfusion deficits seen in M1 and ICA territory occlusions may appear similar in many cases, though those displayed here best demonstrate the easily differentiable pattern archetypes. our study. We did not include patient outcome as an outcome measure of this study given the heterogeneous population studied, and, therefore, our study is unable to measure how changes in neuroimaging protocols may impact patient outcome.

\section{CONCLUSIONS}

Patients with anterior circulation AIS due to a LVO are accurately identified on DWI and PWI alone, and the information provided by MRA may be correctly inferred from PWI. Omission of MRA may be considered to expedite AIS imaging and treatment in appropriate clinical scenarios or may be confidently supplanted if non-diagnostic or artifact-limited.

Contributors All authors made substantial contributions to the conception and design of the study, acquisition of data, or analysis and interpretation of data.

Funding The authors have not declared a specific grant for this research from any funding agency in the public, commercial, or not-for-profit sectors.

Competing interests Gregory Albers is a shareholder of ischemaView.

Patient consent Detail has been removed from this case description/these case descriptions to ensure anonymity. The editors and reviewers have seen the detailed information available and are satisfied that the information backs up the case the authors are making.

Ethics approval Stanford University Institutional Review Board.

Provenance and peer review Not commissioned; externally peer reviewed.

Data sharing statement Data sharing. Published and unpublished data may be shared for additional publications pending the authors' agreement to a research collaboration when appropriate.

(c) Article author(s) (or their employer(s) unless otherwise stated in the text of the article) 2018. All rights reserved. No commercial use is permitted unless otherwise expressly granted.

\section{REFERENCES}

1 Mattioni A, Cenciarelli S, Biessels G, et al. Prevalence of intracranial large artery stenosis and occlusion in patients with acute ischaemic stroke or TIA. Neurol Sci 2014;35:349-55

2 Patel VP, Heit JJ. Ischemic stroke treatment trials: neuroimaging advancements and implications. Top Magn Reson Imaging 2017;26:133-9.

3 Khatri P, Yeatts SD, Mazighi M, et al. Time to angiographic reperfusion and clinical outcome after acute ischaemic stroke: an analysis of data from the Interventional Management of Stroke (IMS III) phase 3 trial. Lancet Neurol 2014;13:567-74.

4 Warach SJ, Luby M, Albers GW, et al. Acute stroke imaging research roadmap III imaging selection and outcomes in acute stroke reperfusion clinical trials: consensus recommendations and further research priorities. Stroke 2016:47:1389-98.

5 McTaggart RA, Ansari SA, Goyal M, et al. Initial hospital management of patients with emergent large vessel occlusion (ELVO): report of the standards and guidelines committee of the Society of Neurolnterventional Surgery. J Neurointerv Surg 2017:9:316-23.

6 Wahlgren N, Moreira T, Michel P, et al. Mechanical thrombectomy in acute ischemic stroke: consensus statement by ESO-Karolinska Stroke Update 2014/2015, supported by ESO, ESMINT, ESNR, and EAN. Int J Stroke 2016;11:134-47.

7 Albers GW, Thijs VN, Wechsler L, et al. Magnetic resonance imaging profiles predict clinical response to early reperfusion: the diffusion and perfusion imaging evaluation for understanding stroke evolution (DEFUSE) study. Ann Neurol 2006;60:508-17. 
8 Lansberg MG, Straka M, Kemp S, et al. MRI profile and response to endovascular reperfusion after stroke (DEFUSE 2): a prospective cohort study. Lancet Neurol 2012;11:860-7.

9 Menjot de Champfleur N, Saver JL, Goyal M, et al. Efficacy of stent-retriever thrombectomy in magnetic resonance imaging versus computed tomographic perfusion-selected patients in SWIFT PRIME Trial (Solitaire FR With the Intention for Thrombectomy as Primary Endovascular Treatment for Acute Ischemic Stroke). Stroke 2017:48:1560-6.

10 McTaggart RA, Yaghi S, Sacchetti DC, et al. Mechanical embolectomy for acute ischemic stroke beyond six hours from symptom onset using MRI based perfusion imaging. J Neuro/ Sci 2017;375:395-400.

11 Thomalla G, Boutitie F, Fiebach JB, et al. Stroke with unknown time of symptom onset: baseline clinical and magnetic resonance imaging data of the first thousand patients in WAKE-UP (Efficacy and Safety of MRI-Based Thrombolysis in Wake-Up Stroke: A Randomized, Doubleblind, Placebo-Controlled Trial). Stroke 2017:48:770-3

12 Nogueira RG, Jadhav AP, Haussen DC, et al. Thrombectomy 6 to 24 hours after stroke with a mismatch between deficit and infarct. N Engl J Med 2018;378:11-21.

13 Albers GW, Marks MP, Kemp S, et al. Thrombectomy for stroke at 6 to 16 hours with selection by perfusion imaging. N Engl J Med 2018;378:708-18.

14 Marks MP, Lansberg MG, Mlynash M, et al. Angiographic outcome of endovascular stroke therapy correlated with MR findings, infarct growth, and clinical outcome in the DEFUSE 2 trial. Int J Stroke 2014;9:860-5.

15 Simonsen CZ, Madsen MH, Schmitz ML, et al. Sensitivity of diffusion- and perfusion-weighted imaging for diagnosing acute ischemic stroke is $97.5 \%$. Stroke 2015;46:98-101.

16 Vagal A, Menon BK, Foster LD, et al. Association between CT angiogram collaterals and CT perfusion in the Interventional Management of Stroke III Trial. Stroke 2016:47:535-8.

17 Souza LC, Yoo AJ, Chaudhry ZA, et al. Malignant CTA collateral profile is highly specific for large admission DWI infarct core and poor outcome in acute stroke. AJNR Am J Neuroradiol 2012;33:1331-6.

18 Liebeskind DS, Tomsick TA, Foster LD, et al. Collaterals at angiography and outcomes in the Interventional Management of Stroke (IMS) III trial. Stroke 2014;45:759-64.
19 Heit JJ, Wintermark M. Imaging selection for reperfusion therapy in acute ischemic stroke. Curr Treat Options Neurol 2015:17:332.

20 Copen WA, Rezai Gharai L, Barak ER, et al. Existence of the diffusion-perfusion mismatch within 24 hours after onset of acute stroke: dependence on proximal arterial occlusion. Radiology 2009;250:878-86.

21 Straka M, Albers GW, Bammer R. Real-time diffusion-perfusion mismatch analysis in acute stroke. J Magn Reson Imaging 2010;32:1024-37.

22 Staroselskaya IA, Chaves C, Silver B, et al. Relationship between magnetic resonance arterial patency and perfusion-diffusion mismatch in acute ischemic stroke and its potential clinical use. Arch Neurol 2001;58:1069-74.

23 Campbell BC, Mitchell PJ, Kleinig TJ, et al. Endovascular therapy for ischemic stroke with perfusion-imaging selection. N Engl J Med 2015;372:1009-18.

24 Saver JL, Goyal M, Bonafe A, et al. Stent-retriever thrombectomy after intravenous t-PA vs. t-PA alone in stroke. N Eng/ J Med 2015;372:2285-95.

25 González RG, Copen WA, Schaefer PW, et al. The Massachusetts General Hospital acute stroke imaging algorithm: an experience and evidence based approach. $J$ Neurointerv Surg 2013;5(Suppl 1):i7-12.

26 Asif KS, Lazzaro MA, Zaidat 0 . Identifying delays to mechanical thrombectomy for acute stroke: onset to door and door to clot times. J Neurointerv Surg 2014;6:505-10.

27 Menon BK, Sajobi TT, Zhang Y, et al. Analysis of workflow and time to treatment on thrombectomy outcome in the Endovascular Treatment for Small Core and Proximal Occlusion Ischemic Stroke (ESCAPE) Randomized, Controlled Trial. Circulation 2016:133:2279-86.

28 Liebeskind DS, Jahan R, Nogueira RG, et al. Early arrival at the emergency department is associated with better collaterals, smaller established infarcts and better clinical outcomes with endovascular stroke therapy: SWIFT study. J Neurointerv Surg 2016:8:553-8

29 Goyal M, Menon BK, van Zwam WH, et al. Endovascular thrombectomy after large-vessel ischaemic stroke: a meta-analysis of individual patient data from five randomised trials. Lancet 2016;387:1723-31.

30 Boulouis G, Siddiqui KA, Lauer A, et al. Immediate vascular imaging needed for efficient triage of patients with acute ischemic stroke initially admitted to nonthrombectomy centers. Stroke 2017;48:2297-300 